\title{
EL LIBRO DE JUDIT: HISTORIA, LITERATURA Y TEOLOGÍA ${ }^{\mathbf{1}}$
}

The book of Judith: history, literature and theology ${ }^{2}$

\author{
Dr. Adolfo D. Roitman \\ Curador Rollos del Mar Muerto \\ Santuario del Libro, Museo de Israel \\ shrine@imj.org.il
}

\section{Resumen}

El libro de Judit es un libro apócrifo judío, escrito probablemente para finales del siglo II a.e.c. en la tierra de Israel, y existente solamente en versión griega o en versiones hechas a partir de ésta. La obra de carácter novelesco cuenta sobre la proeza de Judith, una mujer piadosa, bella, viuda y rica, que con arrojo y picardía decapitó al general Holofernes, salvando así a los judíos de Betulia de caer en manos de los asirios. La obra presenta un plan estructural muy complejo (una pirámide quiástica piramidal), el cual sirve como soporte literario para comunicar una doctrina religiosa original, a saber: una revisión de la tradicional doctrina de la retribución. El objetivo de esta composición habría sido presentar de una manera novelada una reflexión en retrospectiva acerca del significado religioso de la revuelta macabea.

Palabras clave: Libro de Judit - Historia - Literatura -Teología.

\begin{abstract}
The Book of Judith is a Jewish apocryphal book, probably written by the late second century BCE in the land of Israel, and existing only in Greek version or in versions made from it. The work of fictional character tells of the prowess of Judith, a pious, beautiful, rich widow woman, who with courage and mischief beheaded general Holofernes, saving the Jews of Bethulia fell into the hands of the Assyrians. The work presents a very complex structure plan (a pyramid chiastic pyramid), which serves as a literary support for

1 Este artículo es la versión española del trabajo publicado en inglés en el catálogo de la exhibición llamada Heroine of a Thousand Pieces: the Judith Mosaics of Lilian Broca (Vancouver; Il Museo at the italian Culture Centre, 2015), pp. 3-10.

2 Publicado en Cuadernos Judaicos - ISSN: 0718-8749 № 32 Diciembre 2015237
\end{abstract}


communicating an original religious doctrine, namely: a review of the traditional doctrine of retribution. The objective of this composition might have been to present in a fictionalized way a reflection in hindsight about the religious significance of the Maccabean revolt.

Keywords: Book of Judith - History - Literature - Theology.

El libro de Judit es un libro apócrifo (no canónico o sagrado) para judíos y protestantes, pero deuterocanónico (inspirado) para los católicos (según lo fijado por el Concilio de Trento [1545-1563]: "verdadera y auténtica palabra de Dios"). No hay unanimidad en cuanto a la ubicación del mismo en los manuscritos. En la versión griega Alejandrina y en la latina de la Vulgata, Judit está entre Tobías y Ester; en la versión griega del Vaticano está entre Tobías y la Sabiduría de Salomón; mientras que Lutero la ubica al comienzo de los libros apócrifos.

\section{Contenido}

La trama del relato gira en derredor de una piadosa mujer llamada Judit, quien con osadía, valor y astucia salvó al pueblo de Israel de caer en manos de los asirios, comandados por el general Holofernes.

La historia comienza en el año doce del reinado de Nabucodonosor, que reinaba sobre los asirios en la gran ciudad de Nínive, cuando el rey de los medos Arfaxad fortificó su capital Ecbatana. Al considerar este hecho como una acción de rebeldía, Nabucodonosor convocó a los pueblos de su imperio, desde Elam y Persia hasta los confines de Egipto y Etiopía, para sofocar juntos este conato de sublevación. A pesar de que todos los pueblos de occidente (Asia Menor, Mesopotamia, Siria, la tierra de Israel y Egipto) desoyeron este llamado, el rey asirio destruyó Ecbatana sin otra ayuda que las tropas orientales, prometiendo vengarse de los pueblos desleales (cap. 1).

En el año dieciocho de su reinado, el rey Nabucodonosor llamó a Holofernes, jefe supremo del ejército y segundo suyo, para ordenarle salir al frente de un ejército con el propósito de 
castigar a los pueblos rebeldes. Sin demora, una fuerza militar descomunal salió de Nínive, conquistando exitosamente y sin contratiempos los pueblos occidentales del imperio, hasta finalmente llegar a la tierra de Israel y acampar frente a la llanura de Esdrelón (caps. 2-3). Como era de esperar, el pánico cundió entre los israelitas, temiendo por la suerte de Jerusalén y el Templo. Pero en vez de caer en la desesperanza, el Sumo Sacerdote envió a las fronteras la orden de obstruir los desfiladeros, especialmente en Betulia, suplicando a Dios con ayunos y rogativa solemnes que no entregue la casa de Israel a los impíos (cap. 4).

Sorprendido ante esta inusual reacción por parte de los judíos, Holofernes convocó a su consejo de guerra en el campamento para conocer detalles sobre este pueblo desconocido. Entonces Ajior, el jefe de los amonitas, después de pasar revista a la historia del pueblo, le advirtió a Holofernes no atacar a Israel, ya que su historia prueba que son invencibles mientras permanecen fieles a su Dios. Como consecuencia de estas desafiantes palabras, Ajior fue expulsado del campamento, para luego ser capturado por los israelitas y traído como prisionero a Betulia. Al otro día, y desoyendo las advertencias de Ajior, Holofernes puso sitio a la ciudad. Después de treinta y cuatro días de asedio, el hambre y la sed quebraron la resistencia de los habitantes, exigiendo a Ozías y a los otros jefes de la ciudad rendirse ante el enemigo. En respuesta a esta petición, Ozías pidió un plazo extra de cinco días, esperando que durante este tiempo Dios mostraría su compasión hacia Israel (caps. 57).

En este momento dramático del relato aparece por primera vez en escena la heroína Judit, una mujer viuda, bella, sabia y piadosa. Después de reprocharle a Ozías y a los ancianos por su falta de fe en Dios, ella les anunció su decisión de salvar a Israel, sin detallar su plan de acción. Después de orar a Dios solicitando su ayuda, Judit se quitó sus ropas y se bañó, para luego vestirse y adornarse con lo mejor de su ajuar. Acicalada y con una vianda de comida ritualmente pura, Judit y su sierva salieron en la noche de la ciudad para dirigirse al campamento asirio (caps. 7-10).

Su belleza y astucia le abrieron el paso hasta la tienda del jefe Holofernes, consiguiendo quedarse en el campamento, con la promesa de que Dios le comunicaría cuándo y cómo 
podría atacar a Israel. A pesar de residir en medio de los paganos, Judit se mantuvo fiel a Dios. Cada noche se dirigía a la fuente fuera del campamento para purificarse, y durante el día no tocaba los alimentos impuros, alimentándose con las provisiones que había llevado consigo (cap. 11)

Al cuarto día el general Holofernes la invitó a un banquete con el claro propósito de seducirla y violarla. Pero fue precisamente durante esa noche decisiva cuando la suerte de los personajes cambió de manera imprevista. Al quedar Holofernes y Judit solos en la tienda después de que todos los participantes se retiraron al final del festín, la heroína judía decapitó al general asirio, aprovechando que éste yacía desplomado sobre el lecho por el sopor producido por el vino. Logrado el objetivo, Judit y su sirvienta salieron como todas las noches del campamento, pero esta vez ya no para purificarse, sino para volver a Betulia con la cabeza de Holofernes en el saco de provisiones de su sirvienta. Una vez de regreso, Judit contó su hazaña a sus conciudadanos, mostrándoles la cabeza de Holofernes (caps. 12-13).

Impresionado a la vista de la cabeza decapitada del asirio y sorprendido por las buenas nuevas, Ajior confesó su fe en el Dios de Israel y se hizo circuncidar al momento. Al descubrir lo sucedido, el enemigo huyó a la desbandada; Israel acudió de todas partes para saquear los despojos. El Sumo Sacerdote acudió en persona desde Jerusalén para felicitar a la heroína. En acción de gracias, Judit se dirigió en procesión a Jerusalén para ofrecer en el Templo los despojos del enemigo, entonando en esa oportunidad un canto de victoria. A su regreso a Betulia, Judit retomó su vida anterior entregada a la viudez, el ayuno y la oración, rehusando hasta la muerte todos los partidos que se le ofrecieron para casarse (caps. 14-16).

\section{Lengua original y versiones}

La mayoría de los estudiosos considera que el texto griego de Judit (existente en cuatro códices unciales de la traducción de los Setenta [siglos IV-V]) $)^{3}$ es la versión de una obra

3 La traducción de los Setenta o Septuaginta es la traducción de la Biblia Hebrea al griego, realizada por los judíos en Alejandría entre los siglos III-I a.e.c 
escrita originalmente en una lengua semítica, probablemente en hebreo, dados los muchos hebraísmos y los errores de traducción del hebreo. ${ }^{4}$ Sin embargo, ningún fragmento de esta obra ha sido hallado entre los rollos del Mar Muerto, ${ }^{5}$ como así tampoco ningún texto hebreo antiguo nos ha llegado a nuestras manos. ${ }^{6}$

Según el testimonio del renombrado Padre de la Iglesia Jerónimo (c. 347-420), su versión latina de la Vulgata fue hecha a partir de un texto arameo ("in verbis Chaldaeis"), probablemente derivada a su vez de un texto hebreo o, tal vez, de la versión griega. ${ }^{7}$ Asimismo, se conocen versiones de Judit en copto, siríaco, etíope y armenio, todas hechas a partir de la traducción de los Setenta.

Existen igualmente tres importantes textos hebreos medievales. A pesar de haber sido defendida su originalidad (A. M. Dubarle), arguyendo que estas versiones estarían próximas a la versión aramea utilizada por Jerónimo en su traducción, la mayoría de los estudiosos creen que ellas habrían estado basadas en la versión latina de la Vulgata. Finalmente, se conocen a partir de los siglos X y XI unas doce adaptaciones tardías de esta leyenda en desarrollos exegéticos (midrashim) y poemas litúrgicos (piyyutim) medievales.

\section{Género literario}

El libro de Judit pretende ser una obra de carácter histórico, que relata sobre las proezas de una heroína del lejano pasado de Israel. En este sentido, el texto en cuestión parece

4 Sin embargo, la hipótesis sobre una redacción original en griego del libro ha tomado fuerza en lo últimos años.

5 Estos manuscritos judíos antiguos fueron hallados entre los años 1947-1956, en 11 cuevas de la zona del Mar Muerto, próximas a las ruinas de Qumrán (un probable asentamiento de la secta esenia).

6 Ninguna referencia de esta historia se halla presente en las obras del filósofo judío Filón de Alejandría (20 a.e.c.?-o 50 e.c.?), el historiador jerosolimitano Flavio Josefo (37?-100 e.c?) o en los escritos rabínicos anteriores a la época medieval (siglo X), a excepción de la referencia a la historia de Judit presente en la homilía de Rav Ahai (680-c.e) (no es del toda segura la originalidad de ésta última). La primera referencia a la historia de Judit aparece en una obra de los padres apostólicos (Epístola de Clemente a los Corintos 1.55 [siglo I-II e.c.]). Asimismo cabría la posibilidad de que el relato sobre el asesinato de Sísara por Yael (cap. 31) presente en la obra pseudoepigráfica conocida como Antigüedades Judías o Pseudo-Filón (¿siglo I e.c.?) habría sido modelada según el relato sobre el asesinato de Holofernes por Judit.

7 Lo que probaría, que a pesar de la ausencia de toda referencia a la historia de Judit en las fuentes judías antiguas, este relato habría sido conocido en círculos populares de la época. 
transpirar credibilidad al cumplir todos los requisitos de cualquier obra histórica: fechas; nombres de lugares y personajes históricos (algunos conocidos y otros desconocidos); descripciones de eventos reales, sin referencia alguna a intervenciones sobrenaturales propias de las leyendas. Sin embargo, las contradicciones, las imprecisiones, los errores e improbabilidades flagrantes en el campo de la historia y la geografía ${ }^{8}$ como así también la combinación de varios relatos y personajes bíblicos, ${ }^{9}$ ponen en duda cierta el carácter "histórico" de la obra. ${ }^{10}$

A la luz de lo expuesto más arriba, entonces, los investigadores modernos han llegado a la conclusión de que el relato de Judit sería una ficción, habiendo propuesto distintos géneros literarios para definir su carácter, a saber: "novela histórica"; "relato didáctico-moral"; "alegoría"; "novela política", "leyenda-apocalipsis", y otros. Un avance significativo en este sentido fue hecho por algunos estudiosos al resaltar el carácter imaginario (J. Licht) y paradigmático del relato (freie parabolische Geschichtsdarstellung, según E. Haag), ${ }^{11}$ definiendo la obra como una "novela teológica" (E. Zenger), con rasgos típicos propios de relatos tradicionales-folclóricos (C. Moore; M. P. Coote).

\section{Fecha y lugar de composición}

Si una manera aceptada de datar una obra es tomar en consideración los detalles presentes en la misma - como ser: nombres de personas y lugares; términos técnicos; costumbres;

\footnotetext{
${ }^{8}$ Entre las numerosas contradicciones históricas se puede mencionar a título de ejemplo el hecho de que el histórico Nabucodonosor (605-561 a.e.c.) fue un rey babilónico y no asirio, y su capital Nínive fue destruida en el año 612 a.e.c., años antes de subir éste al trono. Y como ejemplo de las improbabilidades geográficas se puede destacar que la ciudad de Betulia, a pesar de los datos ofrecidos en la obra, no es mencionada por ninguna otra fuente. Y de aquí la conclusión, que esta ciudad habría sido fruto de la imaginación del autor.

${ }^{9}$ Por ejemplo, la historia de Judit combina la decapitación de Goliat por medio de David (1 Samuel 17) con el asesinato de Sísara por Yael (Jueces 4).

${ }^{10}$ Sin embargo, la mayoría de los investigadores católicos, como así también algunos estudiosos judíos y protestantes, han tratado de sortear sin éxito estas dificultades geográficas e históricas por medio de artilugios rebuscados.

${ }^{11}$ La figura de Judit (en hebreo, "la Judía") está lejos de representar una figura real de carne y hueso, con virtudes y defectos. Todas sus cualidades (pedigree, belleza, riqueza, piedad, sabiduría, valentía y astucia) la convierten ciertamente en un modelo paradigmático sublime y perfecto, digno de emular por Israel. Como lo decía el mismo autor: "era muy bella y muy parecida [...] No había nadie que pudiera decir de ella una palabra maliciosa, porque era muy temerosa de Dios" (8, 8 [Biblia de Jerusalén, Bilbao 1975]).
} 
o alusiones a eventos históricos conocidos-, pues en el caso de nuestra obra, la aplicación de esta metodología es bastante problemática. Ya que una característica notable del libro de Judit es que el mismo presenta detalles que corresponderían a dos épocas distintas: persa y helenística. ${ }^{12} \mathrm{El}$ consenso entre los investigadores es que el último estadio de redacción de la obra, si no la composición toda, habría acaecido en la época de los Macabeos-Asmoneos, probablemente en el último tercio del siglo II a.e.c.

Como es de esperar, tampoco el lugar de la composición ha recibido una respuesta unánime. Tanto sea por el conocimiento que el autor muestra en el campo de la geografía, o sea por la probable composición original de la obra en hebreo, la mayoría de los estudiosos cree que el libro habría sido compuesto en la tierra de Israel. ${ }^{13}$

\section{Estructura y significado teológico}

La obra presenta un plan literario estructural muy complejo. A los efectos de resolver los problemas literarios de la obra, ${ }^{14}$ se han propuesto dos modelos complementarios (A. Roitman). El primer modelo es una estructura piramidal, según la cual la trama del libro puede dividirse en cinco partes: 1) exposición $(1,1-4,15) ; 2)$ inicio de la acción $(5,1-7$, 18); 3) clímax $(7,19-9,14)$; 4) caída de la acción $(10,1-14,10)$; 5) final feliz $(14,11-$ 16, 25). ${ }^{15} \mathrm{El}$ segundo modelo es una estructura quiástica piramidal, ${ }^{16}$ cuyo diseño

\footnotetext{
${ }^{12}$ Por un lado, por ejemplo, la onomástica (como en el caso de los nombres de Holofernes y Judit) apunta a la época persa. Pero, por el otro, las alusiones a Nabucodonosor como un rey divinizado $(3,8 ; 6,2)$ concuerdan con la manera en que los textos de la época helenística presentan al rey griego-seleúcida Antíoco IV Epífanes (215-164 a.e.c.). Muy especialmente es notable la presencia en la obra de elementos literarios que indican una clara influencia de las obras apócrifas 1-2 Macabeos, como ser: la exhibición de la cabeza del comandante (Holofernes en Judit 14, 1, 11; Nicanor en 1 Macabeos 7, 47; 2 Macabeos 15, 35).
}

${ }^{13}$ Una minoría ha propuesto como lugares alternativos de composición algunas de las diásporas judías: Egipto, Siria, e incluso, Persia.

${ }^{14}$ Como ser: ¿Por qué hay una disminución en el uso del discurso directo en los primeros cuatro $(2,5-13 ; 3$, $2-4)$ y en los últimos capítulos $(15,9-10 ; 16,1-17)$, mientras que hay un claro aumento de este uso en los capítulos 5-14 ¿Por qué se lo describe a Holofernes de una manera indirecta en los capítulos 2-3, mientras que se lo describe de una manera directa en los capítulos 5-13? ¿Por qué Nabucodonosor aparece solo en los primeros dos capítulos? ¿Por qué Ajior aparece por primera vez en los capítulos 5-6, y luego de una larga ausencia, reaparece en el capítulo 14, 5-10)? ¿Por qué Jerusalén juega un rol fundamental solo en los capítulos 4 y 16 ?

15Este modelo teórico para entender la estructura literaria del libro de Judit a nivel narrativo está basado en los estudios sobre la estructura dramática del teatro griego clásico y shakespereano llevados a cabo por el 
permite identificar un balance simétrico entre las dos mitades de la pirámide. De acuerdo a esto último, cada unidad literaria de la primera mitad es reflejada en la segunda mitad de la pirámide.

Este modelo estructural permite descubrir la existencia de dos temas teológicos, combinados de manera artística en la obra. El primero de ellos es la confrontación entre el paganismo y el monoteísmo. Este conflicto ocupa las partes periféricas de la estructura piramidal (es decir, las secciones 1, 2, 4 y 5), ubicadas a ambos lados del clímax. A diferencia de otros relatos bíblicos, este conflicto no tiene un carácter militar o político, sino antes bien, es de tipo meta-histórico y paradigmático. La verdadera confrontación es entre Yahvé y Anti-Yahvé. Este conflicto general entre estas dos fuerzas espirituales se manifiesta en el relato en tres niveles específicos: 1) nivel metafísico: Nabucodonosor vs. Dios de Israel (1, 1-2, 13; 16, 1-25); 2) nivel nacional: Asiria vs. Israel (2, 14-4, 15; 14, 11 15, 13); y 3) nivel individual: Holofernes vs. Judit (5, 1-7, 18; 10, 1-14, 10).

Pero el mensaje religioso que se deriva de todos estos conflictos particulares es siempre el mismo, a saber: la superioridad de la fe israelita. A decir verdad, esta idea no tiene nada de original; la misma puede ser encontrada en otras fuentes de la tradición bíblica (ver 1 Samuel 17, 45-47). Sin embargo, la originalidad del libro de Judit reside en la sugerencia de que este conflicto puede ser resuelto por medio de la institución de la conversión. Según esta obra, el converso (Ajior) parece la única persona capaz, desde una perspectiva estructuralista, de resolver a manera de "mediador" la tensión binaria existente entre el monoteísmo y el paganismo. Este mensaje universal es una contribución significativa de la obra, pero no su mensaje religioso central.

El segundo tema teológico de la obra tiene que ver con la confrontación entre la fe y la incredulidad, que aparece de manera manifiesta en el clímax de la obra (7, 19-9, 14). La

crítico de teatro alemán G. Freytag (Technik des Dramas, 1863

${ }^{16}$ Este modelo de análisis fue desarrollado originalmente por Y. Raday ("Sobre el quiasmo en la narrativa bíblica", Beth Mikra 20/21 [1963-1964] 48-72 [en hebreo]) para entender la estructura literaria de los relatos bíblicos presentes en la Biblia Hebrea. 
incredulidad es representada por los ciudadanos y los dirigentes de Betulia, quienes creían que el sufrimiento y el dolor eran resultado del castigo divino por los pecados cometidos (7, 28). Su actitud cuasi-herética era fruto de su manera literal de entender la idea retributiva de castigo proclamada por la teología deuteronomista del pacto (Deuteronomio 30, 15-20). Frente a este enfoque que llevaba a la desesperanza y a la herejía, el libro de Judit propone en la cúspide quiástica de la estructura piramidal $(8,11-27)$ una revisión de la tradicional doctrina de la retribución. Según esta nueva interpretación, el sufrimiento y las penas pueden tener un propósito educativo, teniendo un carácter de prueba divina. Por ello, frente a la crisis y el dolor, Israel no debe desesperar sino poner toda su confianza en Él. Sin embargo este mensaje tradicional de fuerte raigambre bíblica (por ejemplo, el libro de Job), tiene en Judit una implicación original. Ya que esta teología no lleva en el libro al fatalismo o a la resistencia pasiva, sino a una postura activista $(8,32)$. Y de aquí la conclusión, pues, que esta nueva interpretación de la antigua teología deuteronomista sería ciertamente el mensaje religioso central de la obra.

En caso de estar acertados en la identificación del tema teológico central del libro, el mismo entonces encuadraría perfectamente en la ideología activista y militarista adoptada por los Macabeos a partir de su revuelta contra las persecuciones religiosas iniciadas por Antíoco IV Epífanes (167 a.e.c.) (1 Macabeos 2, 23-70), y desde entonces promovida por los Asmoneos durante los gobiernos de Simón, Juan Hircano I, y durante los reinados de Aristóbulo I y Alejandro Janeo (segunda mitad del siglo II y primer cuarto del siglo I a.e.c.). Y en caso de ser así, entonces, el objetivo del libro de Judit no habría tenido solo un propósito literario-estético, sino antes bien, presentar de una manera plástica y novelada una reflexión en retrospectiva acerca del significado religioso que la revuelta macabea habría tenido en la historia de Israel para redefinir la antigua y sacrosanta tradición bíblica.

\section{Consideraciones finales}

Esta ideología activista presente en el libro de Judit y su relación casi umbilical con los Macabeos podría también explicar las razones por las cuales esta obra habría caído en el olvido en la civilización judía, hasta casi desaparecer de la conciencia de Israel hasta la 
época medieval. Los rabinos habrían oscurecido y difuminado conscientemente los triunfos militares de los Macabeos después de los fracasos de las revueltas judías contra los romanos (66-73, 132-135e.c.), que habían llevado al pueblo hebreo hasta el borde del precipicio, con el propósito definido de desacreditar las ideologías radicales, militares y mesiánicas por sus resultados deletéreos para la supervivencia del pueblo de Israel. En caso de ser cierta esta suposición, entonces, se podría suponer que estos mismos sabios habrían adoptado también esa misma estrategia en el caso del libro de Judit, por considerarlo contraproducente con el quietismo político adoptado por los rabinos.

Cuando cambiaron las condiciones políticas y sociales en Europa occidental para el siglo X, y la historia de los macabeos hizo su reaparición en la conciencia judía (como lo prueba el caso del libro Yossipon, escrito en hebreo probablemente en el sur de Italia en el siglo X), también el relato de Judit comenzó a popularizarse entre los judíos. Desde entonces, la historia de esta heroína, y especialmente la escena de la decapitación de Holofernes, se convirtió en un tema muy popular en la literatura y liturgia judía, en los manuscritos iluminados, y en el arte judío. Prueba de esto último son los numerosos candelabros de ocho brazos utilizados en la fiesta de las Luminarias o Janucá, en donde aparece representada Judit blandiendo en su mano derecha la espada, y en su mano izquierda sosteniendo de los pelos la cabeza decapitada del general Holofernes.

\section{Bibliografía}

Alonso-Schökel, L., "Narrative Structures in the Book of Judith", en: Protocol of the Colloquy of the Center for Hermeneutical Studies in the Hellenistic and Modern Culture: 11 (March, 1974)(ed. W. Wuellner; Berkeley, Ca.: Center for Hermeneutical Studies, 1975) 1-20.

Celada, B., "Nuevos datos acerca de Judith como leyenda religioa judeo-helenística", Cultura Bíblica 257 (1974) 238-245.

Colunga, A., "El género literario de Judit", Ciencia Tomista 74 (1948) 98-126.

Craven, T., Artistry and Faith in the Book of Judith, SBL Dissertation Series 70 (Chico, Ca.:Scholars Press, 1983). 
Delcor, M., “Le livre de Judith et l'epoque grecque”, Klio 49 (1967) 151-179.

Dubarle, A. M., "Les textes hébreux de Judith et las étapes de la formation du libre", Biblica 70 (1989) 255-266.

Haag, E., "Die besondere literarische Art des Buches Judith and seine theologische Bedeutung", Trier Theologische Zeitschrift 71 (1962) 288-301.

Halpern-Amaru, B., “Judith, Book of”, The Eerdmans Dictionary of Early Judaism (eds. J. J.

Collins y D. C. Harlow; Grand Rapids: Eerdmans, 2010) 855-857.

Levine Gera, D. "The Jewish Textual Traditions", en: The Sword of Judith (eds. K. R. Brine, E.

Ciletti y H. Lähnemann, Open Book Publishers, 2010) 23-39.

Licht, J. "El libro de Judit como una obra de literatura", en: Sefer Baruch Kurzweil Memorial Volume (eds. M. Z. Kaddari, A. Saltman y M. Schwarcz; Ramat Gan: Schoken, 1975) 169-183 (en hebreo).

Mendoza, C., "Judit, Libro de", en: Gran Diccionario Enciclopédico de la Biblia (ed. gral. A. Ropero Berzosa; Editorial Clie, $3^{\text {a }}$ edición, 2014) 1445-1448.

Moore, C. A., Judith, AB 40B (New York: Doubleday \& Comp, 1985).

Roitman, A. R., "Achior in the Book of Judith: His Role and Significance", en: "No One SpokeIll of Her", Essays on Judith (ed. J. C. VandeKam; Atlanta, Ga.: Scholars Press, 1992) 31-45.

Idem, "La estructura y significado del libro de Judith," (Tesis doctoral no publicada, presentada a la Universidad Hebrea de Jerusalén, 1993) (en hebreo).

Zenger, E., "Der Juditroman als Traditionsmodell des Jahweglaubens", Trier Theologische Zeitschrift 83 (1974) 65-80. 